This is the post peer-review accepted manuscript of:

N. Decarli and D. Dardari, (2018) Time Domain Measurements of Signals Backscattered by Wideband RFID Tags. IEEE Transactions on Instrumentation and Measurement, 67(11) 2548-2560. https://doi.org/10.1109/TIM.2018.2824518

The published version is available online at: https://doi: 10.1109/TIM.2018.2824518

(C) 2018 IEEE. Personal use of this material is permitted. Permission from IEEE must be obtained for all other uses, in any current or future media, including reprinting/republishing this material for advertising or promotional purposes, creating new collective works, for resale or redistribution to servers or lists, or reuse of any copyrighted component of this work in other works 


\title{
Time Domain Measurements of Signals Backscattered by Wideband RFID Tags
}

\author{
Nicolò Decarli Member, IEEE, and Davide Dardari Senior Member, IEEE
}

\begin{abstract}
Passive wideband RFID is increasing interest for its capability of providing high-accuracy tag localization in addition to identification and tag-reader communication. The measurement of backscattering capabilities of wideband antennas is usually conducted in the frequency domain by using network analyzers. In this paper we present a novel setup for time domain measurements of signals backscattered by wideband RFID tags; the acquired time domain signal samples, were the backscattered signal (i.e., the antenna mode) is not directly observable, are processed with the proposed scheme in order to extract the component of interest. Experimental evaluations are presented for comparing different wideband antennas and show the effects of the setup characteristics and of the processing schemes on the achievable measurement results.
\end{abstract}

Index Terms-Backscatter, RFID, UWB, Tag, Antenna Mode.

\section{INTRODUCTION}

$\mathbf{I}$ $\mathrm{N}$ the last years, radio-frequency identification (RFID) has increased interest for the fast and efficient identification of goods in warehouses, retail, supply chain and logistic [1]. The introduction of the ultra-high frequency (UHF) tags, with a standard capable of making devices inter-operable [2], has supported the wide spreading of such a radio technique for its capability of offering enhanced services with respect to conventional bar-code based solutions.

More recently, the demand of precise localization of passive RFID tags came to the stage, in order to enable positionbased services capable of making more and more efficient industrial plants and automatic warehouses, preventing the use of other active and energy-hungry solutions resorting to real-time locating systems (RTLS) currently available on the market [3]. Unfortunately, classical narrowband RFID tags are not intended for localization, so the accuracy that can be achieved with these devices is limited [4]. For this reason, the use of new radio techniques implying large bandwidth has been proposed for RFID systems capable of precise localization $[5]-[9]$. In fact, it is well known that the use of signals with large bandwidth, for example impulse radio (IR) ultra-wideband (UWB) signals, enables the accurate ranging,

Manuscript submitted Nov. 7, 2017. Revised Jan. 31, 2018 and Mar. 9, 2018. Accepted Mar. 12, 2018. Date of publication Month XX, 2018; date of current version Month YY, 2018. This work has received funding from the EU Horizon 2020 research and innovation programme under the project XCycle (grant no. 635975), and was supported by the Italian Ministerial PRIN project GRETA (Grant 2010WHY5PR) and by the European Space Agency project LOST.

N. Decarli and D. Dardari are with the DEI, University of Bologna, Via Venezia 52, I-47521 Cesena (FC), Italy, Tel.: +39 0547 339223, (e-mail: \{nicolo.decarli, davide.dardari\}@unibo.it)

Color versions of one or more of the figures in this paper are available online at http://ieeexplore.ieee.org.

Digital Object Identifier XXXXXXX that is, accurate distance measurement, which is needed for positioning [10], [11]. The adoption of a large bandwidth poses the problem of measuring the characteristics of the signals backscattered by passive tags, where the incident radio signal is reflected back (modulated) thus enabling low energy consumption solutions.

Time-domain measurements of reflected signals have been carried out in several contexts mostly related to radar systems [12]-[14]. Usually, wideband or UWB transmitters are employed together with high sampling-rate front-ends at receiver side to acquire the signals reflected by the environment. Unfortunately, such an approach cannot be directly considered for RFID tags based on backscatter, since the antenna mode component, modulated by the load connected to the antenna and here considered as backscattered component of interest, is several orders of magnitude lower in amplitude than the structural mode scattering, related to the reflection of the antenna as a physical object (i.e., clutter). A partial solution for performing measurements of wideband RFID backscattering in the time domain has been shown in [15], where a delay line was used to separate the clutter from the antenna mode backscattering. However, this technique is difficult to implement outside an anechoic chamber due to the presence of the rich multipath characteristic of indoor environments, which bury the useful antenna mode component backscattered by the tag.

For the aforementioned reasons, wideband measurements of backscattering characteristics of tag antennas have been carried out in the frequency domain [16]-[18]. Similar techniques have been adopted for the characterization of the backscattering channel in the presence of UWB tags [19]-[21]. The main drawback of frequency domain measurements, exploiting expensive network analyzers, is its realization by spanning a single-tone continuous wave probe signal among the band of interest. This operation requires the tag and the environment to be static for a certain amount of time, and the time-domain signal reconstruction starting from frequency-domain samples. Moreover, high signal-to-noise ratio (SNR) conditions (i.e., low reader-tag range) are mandatory.

In this paper, a setup for time domain measurements of signals backscattered by wideband RFID tags is presented. The acquired time domain signal samples are processed with an ad-hoc scheme to extract the antenna mode component of interest. The proposed scheme considers the modulation of the tag response with a proper spreading code, whose characteristics are exploited by the receiver processing scheme to isolate the antenna mode contribution from the clutter. Specific processing blocks for the acquisition of the spreading code and the compensation of clock jitters between the transmitter and 
the receivers are also proposed, and their effect characterized with experimentation. The main contributions of the work are:

- The investigation of a time domain measurement technique, with does not require background cancellation, as in radar processing schemes, or the adoption of delay lines at tag side;

- The discussion of ad-hoc processing schemes for the extraction of the antenna mode component of interest, including code acquisition and clock jitter compensation;

- The characterization of the impact of setup and signal processing parameters on the obtained results, presenting real measurements using wideband antennas.

The proposed discussion can serve to realize measurements of the backscatter characteristics of wideband tags, and drive the realization of receivers for wideband RFID.

The remainder of the paper is organized as follows: Sec. II revises the wideband backscattering principle, which is the foundation of passive RFID. Section IIII describes the measurement setup, including the adopted instruments, connection techniques, and presents the signals' characterization. In Sec. IV the ad-hoc signal processing adopted for extracting the antenna mode component of interest is discussed. Finally, experimental results for the characterization of the backscattering capabilities of wideband antennas are presented in Sec. $\nabla$. In this section the impact on the achievable results of the processing and measurements parameters is also discussed. Then, Sec. VI concludes the paper.

\section{WIDEBAND BACKSCATTERING PRINCIPLE}

In this section the backscattering principle, which is the foundation of wideband RFID, is revised. Consider a probe transmitted signal of duration $T_{\mathrm{s}}=N_{\mathrm{s}} T_{\mathrm{p}}$, for example an UWB IR pulse sequence, given by

$$
s(t)=\sum_{i=0}^{N_{\mathrm{s}}-1} p\left(t-i T_{\mathrm{p}}\right)
$$

where $N_{\mathrm{s}}$ is the number of transmitted wideband pulses, $T_{\mathrm{p}}$ is the pulse repetition period (PRP) and $p(t)$ is the bandpass transmitted waveform. A wideband tag is composed of an antenna connected to a switch (i.e., a backscatter modulator). The tag switches its backscatter modulator status at each chip time $T_{\mathrm{c}}=T_{\mathrm{p}} N_{\mathrm{pc}}$, where $N_{\mathrm{pc}}$ is the number of pulses composing a chip, according to an antipodal binary code $\left\{c_{n}\right\}$, for $n=0,1, \ldots, N_{\mathrm{c}}-1$ [5]. An example of wideband backscattered signal corresponding to two different antenna load configurations (open/short) is shown in Fig. 11 considering a single wideband pulse. As can be noticed, there is a polarity change in the reflected antenna mode component, which is the only signal component depending on the antenna load, and that can be exploited for tag identification and localization 1 The backscatter modulator signal commanding the switch of the tag can be written as

$$
m(t)=\sum_{i=0}^{N_{\mathrm{s}}-1} \tilde{c}_{i} \cdot \Pi\left(\frac{1}{T_{\mathrm{p}}}\left[t-i T_{\mathrm{p}}\right]\right)
$$

${ }^{1}$ See [17], [18] for a detailed electromagnetic analysis of UWB backscattering and antennas.

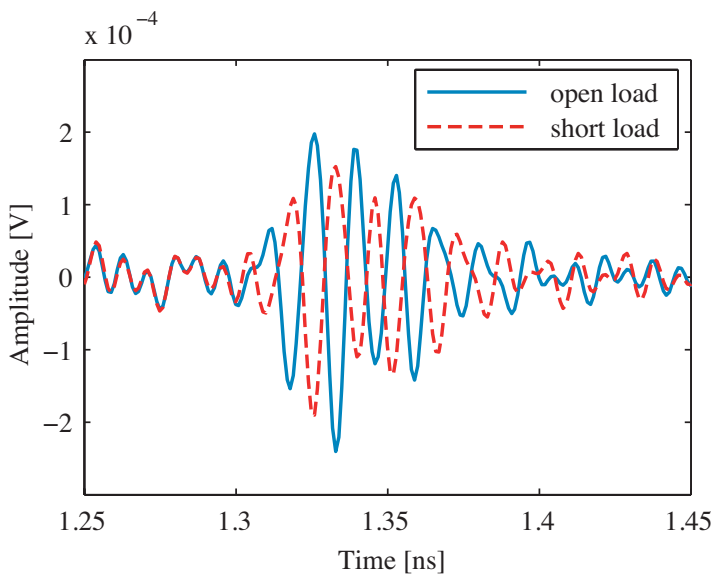

Fig. 1. Example of the backscattered signal for tag open and short circuit loads (only the antenna mode component is shown) [5].

with $\Pi(t)$ denoting the rectangular function of unitary duration for $t \in[0,1]$. In (2) we adopted, for convenience, $\tilde{c}_{i} \triangleq c_{\left\lfloor i / N_{\mathrm{pc}}\right\rfloor}$ for $i=0,1, \ldots, N_{\mathrm{s}}-1$, with $\tilde{c}_{i+N_{\mathrm{s}}}=\tilde{c}_{i}$ In this way, the polarity of the backscattered signal changes at each chip time $T_{\mathrm{c}}$ according to the tag's code value. Notice that, in practical realization of a wideband RFID system, multiple tags can coexist thanks to the adoption of different codes [22]. Other techniques previously proposed include hybrid tags, where the UWB backscatter modulator is activated only when the tag is addressed by another radio interface, such as a standard UHF transponder co-located with the wideband tag.

Each interrogation signal is backscattered by the tag as well as by the surrounding environment. Specifically, the signal at the receiver can be written as

$$
r(t)=r^{\mathcal{T}}(t)+r^{\mathcal{S}}(t)+n(t)
$$

where $r^{\mathcal{T}}(t)$ denotes the tag's contribution, that is, the tag's antenna mode component (comprehensive of multipath in a real scenario), while $r^{\mathcal{S}}(t)$ accounts for the static contribution (comprehensive of multipath) between transmitter and receiver. Finally, $n(t)$ models the additive white Gaussian noise (AWGN). The tag's contribution is given by

$$
r^{\mathcal{T}}(t)=\left[\left(s(t) \otimes h_{\mathrm{down}}(t)\right) \cdot m(t)\right] \otimes h_{\mathrm{up}}(t)
$$

where $h_{\text {down }}(t)$ is the downlink channel impulse response (CIR) related to the link transmitter-tag, $h_{\text {up }}(t)$ is the uplink CIR related to the link tag-receiver. It is possible to rearrange (4) as

$$
r^{\mathcal{T}}(t)=\sum_{i=0}^{N_{\mathrm{s}}-1} \tilde{c}_{i} w^{\mathcal{T}}\left(t-i T_{\mathrm{p}}-\tau\right)
$$

where $\tau$ is the signal time-of-arrival (TOA), considered with respect to the transmitter's clock, and $w^{\mathcal{T}}(t)$ is the channel response to $p(t)$, de-embedded of the propagation time accounted by $\tau$. Specifically, it is $\tau=\left(d_{\mathrm{T}}+d_{\mathrm{R}}\right) / c+\delta$, where $d_{\mathrm{T}}$ is the distance between the transmitter and the tag, $d_{\mathrm{R}}$ is the

\footnotetext{
$2\lfloor\cdot\rfloor$ denotes the floor operation.

${ }^{3}$ Operator $\otimes$ denotes the convolution.
} 


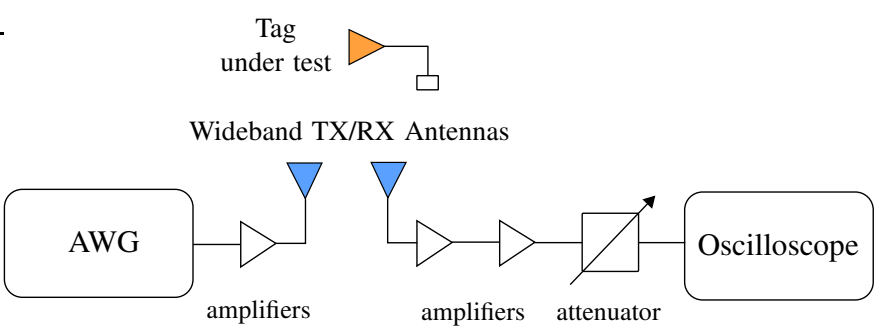

Fig. 2. Schematic representation of the measurement setup.

distance between the tag and the receiver $4 \delta$ models additional time delays (e.g., a delay introduced by the transmission line between the tag antenna and the tag backscatter modulator) and $c$ denotes the speed of light.

Together with the tag response, the receiving antenna collects the signal reflected by the surrounding environment, which composes the static contribution of the clutter component. Such a static contribution $r^{\mathcal{S}}(t)$ in (3) is

$$
r^{\mathcal{S}}(t)=s(t) \otimes h_{\mathrm{c}}(t)
$$

having indicated with $h_{\mathrm{c}}(t)$ the CIR of the link between the transmitter and the receiver, which is independent of tags' backscattering. As before, (6) can be reformulated as

$$
r^{\mathcal{S}}(t)=\sum_{i=0}^{N_{\mathrm{s}}-1} w^{\mathcal{S}}\left(t-i T_{\mathrm{p}}-\tau^{\prime}\right)
$$

where $\tau^{\prime}$ is the clutter signal TOA, considered with respect to the transmitter's clock, and $w^{\mathcal{S}}(t)$ is the channel response to $p(t)$ for the static contribution, de-embedded of the propagation time. Notice that $\tau^{\prime} \approx 0$ when transmitter and receiver are co-located, due to the TX/RX antennas coupling at reader side.

The goal of the measurement system is to characterize the antenna mode component, that is, obtaining an estimate $\widehat{w}^{\mathcal{T}}(t)$ of the backscattered signal $w^{\mathcal{T}}(t)$. However, when conducting measurements, it is $r(t) \approx r^{\mathcal{S}}(t)$, also when in high SNR conditions, since $r^{\mathcal{T}}(t)$ is several orders of magnitude smaller than $r^{\mathcal{S}}(t)$ due to the backscattering mechanism. Then, a proper signal processing is fundamental to extract the antenna mode component from the surrounding static signal and from the noise.

\section{Measurement Setup}

In this section the measurement setup and the adopted instruments and tools are described.

\section{A. Measurement Methodology}

The idea for measuring the backscattering characteristic of wideband antennas in the time domain is to generate signals similar to that adopted by RFID readers according to (1) and collect the environmental response (3) from which the antenna mode component can be extracted. Then, together with the

\footnotetext{
${ }^{4}$ Note that $d_{\mathrm{T}}=d_{\mathrm{R}}$ if the receiver is co-located with the transmitter (i.e., in the case of a monostatic RFID configuration) [23].
}

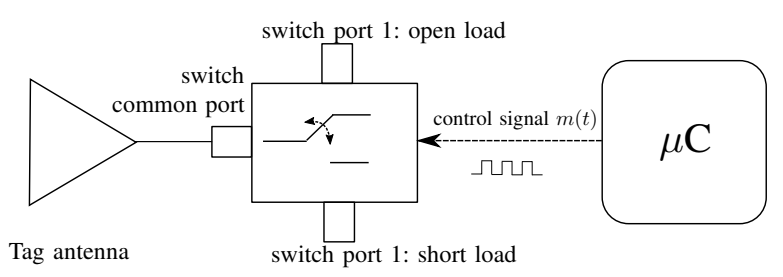

Fig. 3. Tag block schematic.

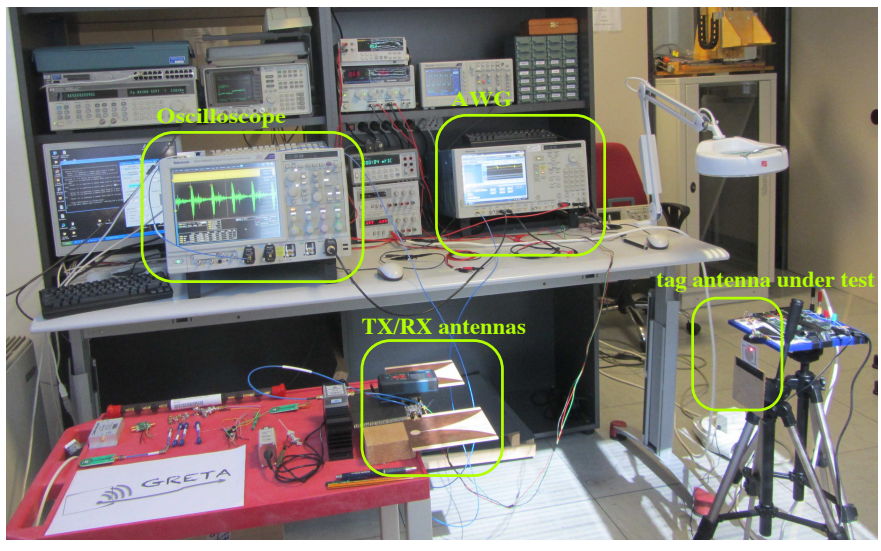

Fig. 4. Picture of the measurement setup.

signal processing described in Sec. IV, the measurement system emulates a RFID receiver.

The measurement setup is shown in Fig. 2] The probe transmitted signal (11) is generated by an arbitrary waveform generator (AWG), amplified with a wideband amplifier and then fed to a wideband transmitting antenna. The transmitted signal propagates in the environment and reaches the tag under test. According to Sec. III it is necessary to implement a strategy to isolate the antenna mode component from the structural mode component, which is part of the clutter. This is possible by changing the load connected to the tag antenna according to the code $\left\{c_{n}\right\}$, as described in (2). Such a task is accomplished by the connection of the tag antenna with a wideband switch, which is loaded with a short circuit load and an open circuit load at its two ports (see Fig. 3). A receiving antenna then collects the signal backscattered by the tag and by the surrounding environment. Such a signal is amplified and acquired by an oscilloscope. A programmable attenuator is inserted between the wideband amplifiers chain and the oscilloscope front-end in order to adapt the instrument dynamic to the amplitude of the incoming signal, preventing the damage of the RF front-end.

A picture of the measurement setup is shown in Fig. 4. In the presented setup the receiving antenna and the transmitting antenna are close each other and form a quasi-monostatic measurement configuration. However, the presented measurement scheme can be applied to different transmitter/receiver antenna configurations, also separating them in space in order to test the backscattering characteristics of tags in bistatic configurations, which are very interesting for RFID applications [24]. The list of instruments, antennas, amplifiers and tools adopted for the presented measurement setup is reported in Tab. 【. 
TABLE I

ADOPTED INSTRUMENTATION.

\begin{tabular}{|c|c|c|c|}
\hline & Component & Producer & Model \\
\hline Transmitter & AWG & Tektronix & AWG7122C \\
Receiver (1) & Oscilloscope (1) & Tektronix & TDS6604 \\
Receiver (2) & Oscilloscope (2) & Tektronix & DPO72304DX \\
Transmitter & Amplifier & Mini Circuits & ZVA-183X-S \\
Receiver & Amplifier (1) & Mini Circuits & ZVA-183X-S \\
Receiver & Amplifier (2) & Mini Circuits & ZVE-8G+ \\
Receiver & Attenuator & Mini Circuits & RCDAT-6000-60 \\
TX/RX & Antennas & Imego & Vivaldi 004 \\
Tag & Switch & Hittite & 104122-5 \\
Tag & Micro controller & Microchip & PIC24FJ256DA210 \\
Tag & Open load & Aeroflex & 7006 \\
Tag & Short load & Aeroflex & 7008 \\
\hline
\end{tabular}

\section{B. Instrumentation and Configuration}

1) Transmitter: The pulse train (11) adopted as probe interrogation signal was built in Matlab according to the IEEE 802.15.4a specifications in terms of central frequency and bandwidth [25]. A root raised cosine (RRC) pulse centered at frequency $f_{\mathrm{c}}=4 \mathrm{GHz}$, with pulse width parameter $T_{\mathrm{w}}=1 \mathrm{~ns}$ and roll-off factor $\beta=0.6$, sampled at frequency $f_{\mathrm{s}}=12 \mathrm{Gs} / \mathrm{s}$ was then imported by the AWG 5 The PRP has been imposed equal to $50 \mathrm{~ns}$; this translates in a maximum reader-tag distance $d_{\max }=2 c T_{\mathrm{p}} \approx 7 \mathrm{~m}$ due to the radio wave traveling time. The amplitude of the transmitted pulse has been set equal to the maximum AWG analog-to-digital converter (ADC) range.

Considering the PRP $T_{\mathrm{p}}=50 \mathrm{~ns}$, the resulting power spectral density (PSD) peak at the instrument output port is $-58 \mathrm{dBm} / \mathrm{MHz}$, corresponding to a transmitted power of $-28.6 \mathrm{dBm}$. In order to have suitable amplitude of the transmitted signal, the AWG output was then connected to wideband amplifier with $26 \mathrm{~dB}$ gain, resulting in a $-32 \mathrm{dBm} / \mathrm{MHz}$ PSD peak ( $9 \mathrm{~dB}$ above the Federal Communications Commission (FCC) limit) 6

2) Receiver: At receiver side, a Vivaldi antenna collects the signal reflected by the environment and the tag. Such a signal is then amplified with a couple of wideband amplifier $(26 \mathrm{~dB}$ and $30 \mathrm{~dB}$ gain, respectively) and fed to an oscilloscope, which operates as a front-end, sampling the incoming signal at frequency $f_{\mathrm{s}}$. Two different oscilloscopes are considered: the first, with $6 \mathrm{GHz}$ bandwidth, operating at $f_{\mathrm{s}}=20 \mathrm{Gs} / \mathrm{s}$ and collecting 200 ksamples (i.e., collecting $10 \mu$ s of the received signal, corresponding to acquire the response to 200 transmitted pulses with $50 \mathrm{~ns}$ PRP); the second, with $23 \mathrm{GHz}$ bandwidth, operating at $f_{\mathrm{s}}=25 \mathrm{Gs} / \mathrm{s}$ and collecting 12,5 Msamples (i.e., collecting $500 \mu \mathrm{s}$ of the received signal, corresponding to acquire the response to 10000 transmitted pulses with $50 \mathrm{~ns}$ PRP). In Sec. $\nabla$ the impact on the measurement quality of the available instrumentation memory, corresponding on the length of the waveform that can be acquired, will be discussed.

Figure 5 presents a comparison between the reference pulse generated by the AWG, and the two pulses obtained at the

\footnotetext{
${ }^{5}$ The instrument has been equipped with an optional board in order to generate UWB signals.

${ }^{6}$ Actually, the FCC limit is imposed on the effective radiated isotropic power (EIRP) so the antenna gain in the maximum direction of the transmitting antenna should be considered.
}

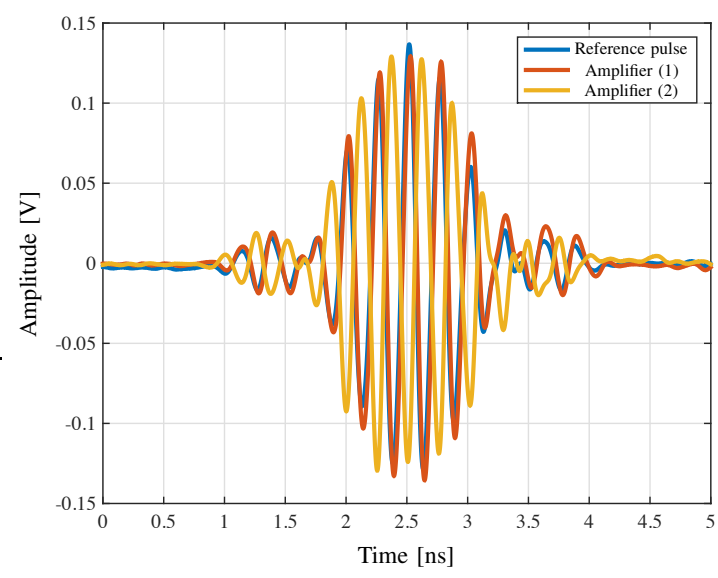

Fig. 5. UWB pulses at the output of different amplifiers (normalized amplitude).

output of the two power amplifiers considered, properly scaled and time-aligned. As it is possible to notice, the distortion introduced by the amplifiers is almost negligible 7 this is confirmed by the cross-correlation coefficients between the reference pulse and the two amplified pulses, whose values are 0.96 for the ZVA-183X-S and -0.91 for the ZVE-8G+.

3) Wideband Tag: The UWB tag has been emulated with discrete components. The tag antenna under test is connected to a wideband switch. Such a switch, with $6 \mathrm{GHz}$ bandwidth, links the antenna port with two SMA ports, depending on the control signal; these two ports are connected to an open and a short circuit load, respectively, as depicted in Fig. 3. The switch is driven by a Microchip DM240312 development board for the PIC24FJ256DA210 micro controller $(\mu \mathrm{C})$. The micro controller was programmed for generating a control signal consisting in a sequence of alternating +1 and -1 chips at different rates, that is, with a variable number of pulses per chip $N_{\mathrm{pc}}$. Then, the resulting codes are, for example:

$$
\begin{aligned}
& \tilde{c}^{(1)}=\{\underbrace{+1,-1}_{\text {alternation of } 1 \text { chip }},+1,-1,+1,-1,+1,-1,+1,-1, \ldots\} \\
& \tilde{c}^{(2)}=\{\underbrace{+1,+1,+1,+1,-1,-1,-1,-1}_{\text {alternation of } 4 \text { chips }},+1,+1,+1,+1,\} .
\end{aligned}
$$

The programmed chip times were $250 \mathrm{~ns}, 500 \mathrm{~ns}, 1 \mu \mathrm{s}, 2 \mu \mathrm{s}$ and $5 \mu$ s (i.e., $N_{\mathrm{pc}}=5, N_{\mathrm{pc}}=10, N_{\mathrm{pc}}=20, N_{\mathrm{pc}}=40$ and $\left.N_{\text {pc }}=100\right)$.

The switch was characterized before its adoption in order to check its performance considering the adoption of wideband signals. A loss of $1.50 \mathrm{~dB}$ and $1.33 \mathrm{~dB}$ was measured between the common port and each of the two input/output ports at the central frequency $f_{\mathrm{c}}=4 \mathrm{GHz} \&$ The distortion introduced by the switch was summarized by means of the crosscorrelation coefficient considering a reference pulse equal to the transmitted one at the common port, obtaining a value

\footnotetext{
${ }^{7}$ Notice that the $30 \mathrm{~dB}$ amplifier ZVE-8G+ introduces a sign inversion of the signal.

${ }^{8}$ Then, due to the backscattering mechanism, the switch loss on the tag signal is about $3 \mathrm{~dB}$ and $2.66 \mathrm{~dB}$, depending on the switch status.
} 


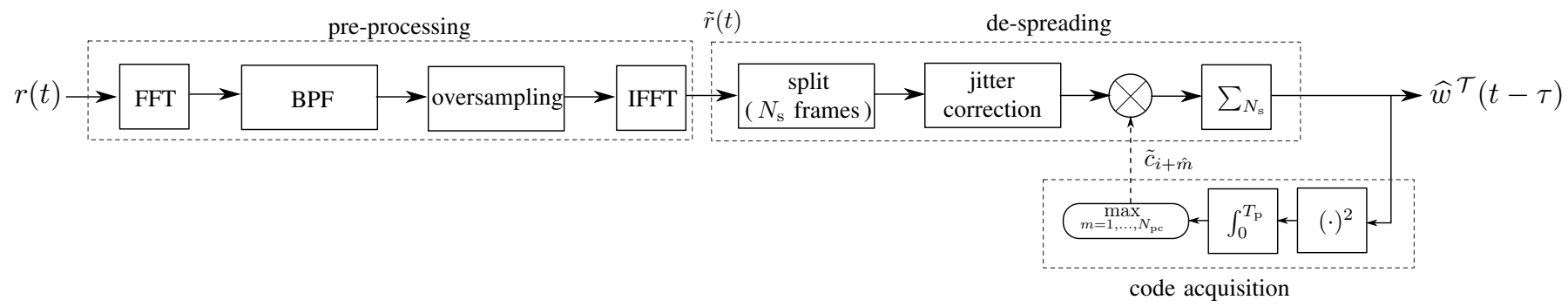

Fig. 6. Schematic representation of the signal processing scheme adopted to extract the antenna mode component of interest.

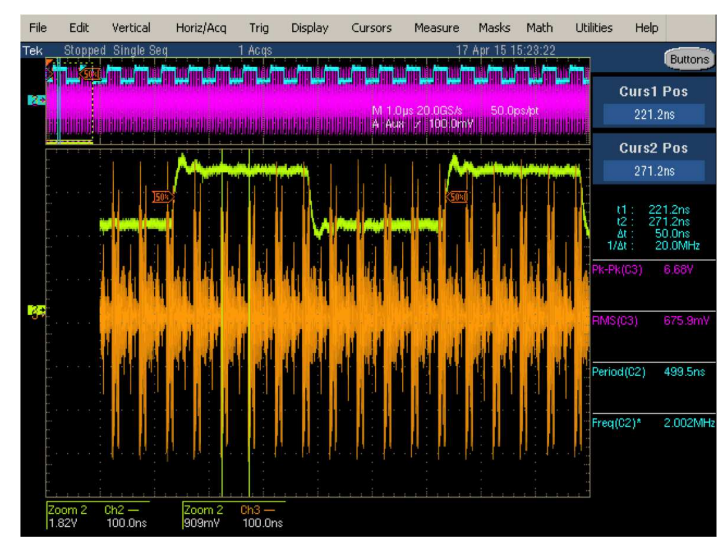

Fig. 7. Example of oscilloscope capture. The orange signal is a zoom of the acquisition composed of the repetition of $N_{\mathrm{s}}$ channel responses to the transmitted pulse. In yellow, a portion of the signal (2) driving the tag switch is reported.

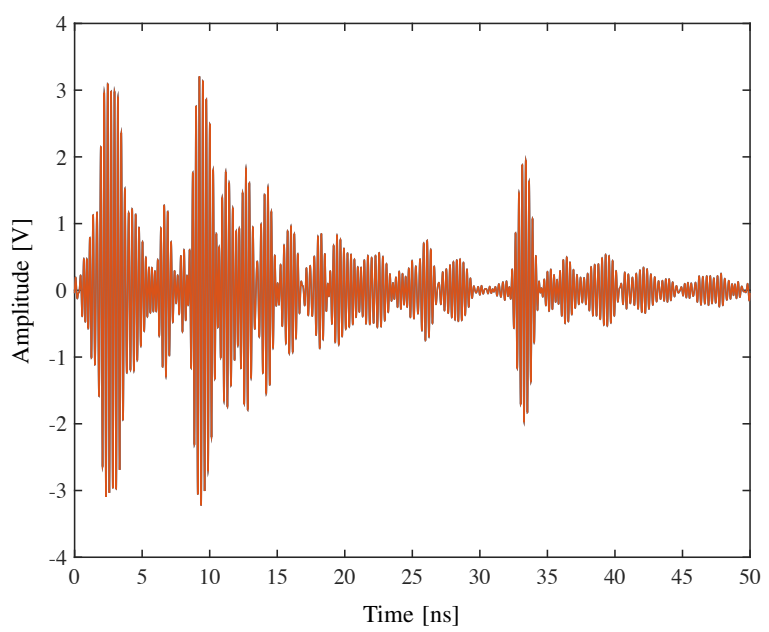

Fig. 8. Measured channel response to a transmitted pulse. The first portion of signal corresponds to the coupling between the transmitting and receiving antenna of the reader.

of 0.88 for both the ports; such a distortion, which could be compensated with an ad-hoc post-processing by characterizing the impulse response of the component, is not considered in the measurement setup since it is characteristic of the tag under measure. Differently, the cross-correlation between the pulses in output at the two ports of the switch was evaluated, since it can impact the measurement setup due to the presence of the de-spreading processing that will be described in Sec. IV the measured correlation coefficient was 0.999 , which ensures the equal behavior of both the ways of the switch, making it suitable for the proposed measurement setup. The delay introduced by the switch was $0.25 \mathrm{~ns}$.

The tag code driving the backscatter modulator at tag side has been considered as free-running and continuously switching the tag status between open and short circuit. The impact of this choice in the processing necessary to extract the antenna mode component will be described in Sec. IV

\section{Signal Acquisition Description}

Figure 7 shows an example of an acquisition where the channel responses to several transmitted pulses are plotted jointly with the tag's switch command signal collected with a probe 9 This measurement was conducted by acquiring the 200 ksamples available from the oscilloscope TDS6604. Figure 8$]$ shows the channel response to a single pulse transmitted by the Vivaldi antenna and collected with the other Vivaldi antenna in the quasi-monostatic configuration. The overall signal $r(t)$ is composed of a series of 200 of these waveforms. The presented waveform encompasses the clutter $r^{\mathcal{S}}(t)$ (i.e., the environment response to the transmitted pulse given by the reflection of the walls, furniture and objects in the room) and the tag response $r^{\mathcal{T}}(t)$ (i.e., the antenna mode). In general, the clutter can be considered almost constant between subsequent frames, while the antenna mode component changes according to the spreading code $\left\{c_{n}\right\}$ driving the switch in the wideband tag. In Fig. 8 it is possible to notice the first large component of the response, that is the coupling between the transmitting and receiving antennas. This is normally the largest contribution and its cancellation is fundamental for a reliable antenna-mode signal characterization. Then, a series of multipath components follow, with different TOA characteristic of the environment where measures are taken and of the antennas' positions and orientations.

\section{Signal Processing}

The goal of the signal processing is to extract, from the raw collected data (3), the backscattered response to a pulse, that is. the tag antenna mode component $w^{\mathcal{T}}(t)$, which is modulated by the switch and then constitutes the useful part. The block diagram of the proposed processing chain is depicted in Fig. 6 . In addition to the measurement goal, the processing scheme can also drive the design of proper algorithm to be adopted

\footnotetext{
${ }^{9}$ It is possible to notice the $250 \mathrm{~ns}$ chip time.
} 

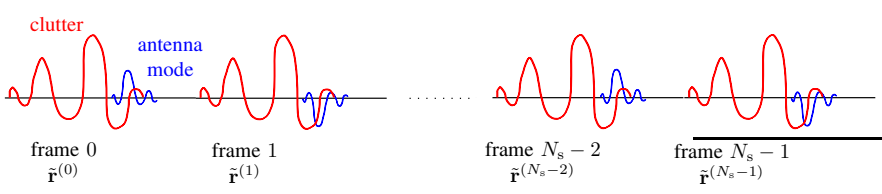

Fig. 9. Signal splitting in $N_{\mathrm{s}}$ frames of length $T_{\mathrm{p}}$.

at reader side in practical implementations. Notice that, the proposed scheme, enables a very simple tag implementation (thus, very low-cost), then moving all the complexity on the reader side, where more powerful hardware can be adopted.

\section{A. Pre-Processing}

First of all, the signal acquired by the oscilloscope is filtered to eliminate the out of band noise. This is realized in the frequency domain, operating a fast Fourier transform (FFT) on the data samples and considering an ideal bandpass filter (BPF) with center frequency $4 \mathrm{GHz}$ and $2 \mathrm{GHz}$ bandwidth. Unfortunately, it has been found experimentally that drift effects, due to the independent local oscillators of the transmitting AWG and receiving oscilloscope, can degrade the measurement capability of the proposed setup. In fact, although the sampling frequency of the oscilloscope is greater than the Nyquist rate, the presence of two different oscillators driving the transmission of the pulses and the acquisition makes the clock skew between the two instruments not negligible, and the effects are particularly pronounced due to the long duration of the transmitted and acquired signal. Moreover, the jitter itself of the oscillator driving the oscilloscope can strongly degrade the clutter removal performance of the successive processing blocks, making impossible the measurement of the backscattered antenna mode signal component. Such effects are then mitigated by operating an oversampling on the receiving signal. This processing operation allows implementing a specific strategy, described in Sec. IV-B, to counteract these clock-related non-idealities of the measurement setup. The effect of different oversampling factors will be characterized in Sec. $\nabla$ In order to reduce the elaboration time, oversampling has been realized in the frequency domain, by inserting a zero-padding sequence in the vector resulting from the filtered signal. Specifically, considering the vector of samples acquired by the oscilloscope $\mathbf{r}=r(n \delta t)$, with $n=1, \ldots, N=T_{\mathrm{s}} / \delta t$ and $\delta t=1 / f_{\mathrm{s}}$, define $\mathbf{s}=\mathrm{FFT}[\mathbf{r}]$. Then, imposing an oversampling factor $\mathrm{OF}$, the time domain oversampled signal $\tilde{\mathbf{r}}=\tilde{r}(n \tilde{\delta} t), \tilde{\delta} t=\delta t / \mathrm{OF}$, of length $N \cdot \mathrm{OF}$ is obtained as

$$
\tilde{\mathbf{r}}=\mathrm{OF} \cdot \operatorname{IFFT}[\tilde{\mathbf{s}}\left(1: \frac{N}{2}\right) \underbrace{0, \ldots, 0}_{N \cdot(\mathrm{OF}-1)} \tilde{\mathbf{s}}\left(\frac{N}{2}: N\right)]
$$

where $\tilde{\mathbf{s}}$ denotes the filtered version of $\mathbf{s}$.

\section{B. De-Spreading}

The core processing enabling the cancellation of the clutter component $r^{\mathcal{S}}(t)$ is the de-spreading. The waveform is split in frames of length equal to the PRP $T_{\mathrm{p}}$ (see Fig. Q), which are multiplied for a code equivalent to that adopted at tag

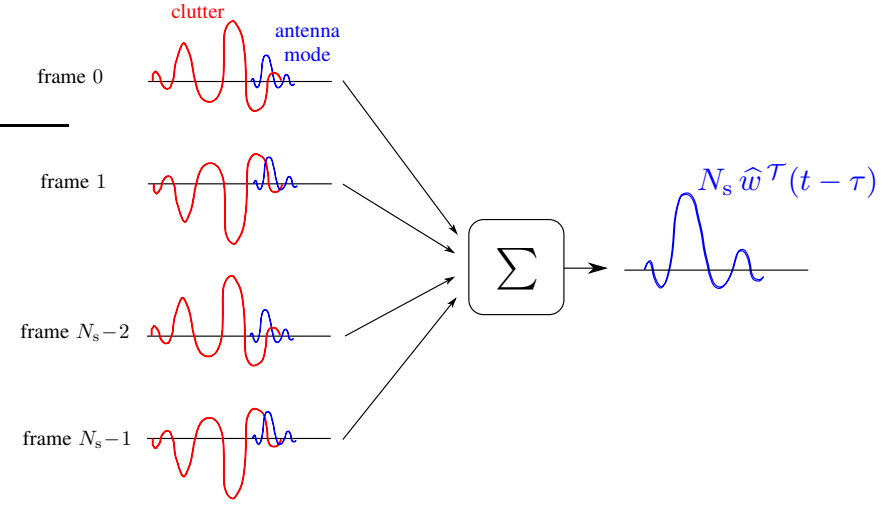

Fig. 10. Pictorial representation of the clutter cancellation principle. Note that the depicted signal portions (of length $T_{\mathrm{p}}$ ) are already multiplied for the tag code.

side. This is possible since the spreading code generated by the micro controller board is known. Then, these frames are summed up together. Formalizing, we define the frame $\tilde{\mathbf{r}}^{(i)}$, for $i=0, \ldots, N_{\mathrm{s}}-1$, as

$$
\tilde{\mathbf{r}}^{(i)} \triangleq \tilde{\mathbf{r}}\left(\left(i \cdot N_{\mathrm{p}}+1\right):\left(i \cdot N_{\mathrm{p}}+N_{\mathrm{p}}\right)\right) \in \mathbb{R}^{N_{\mathrm{p}}}
$$

where $N_{\mathrm{p}}=T_{\mathrm{p}} / \tilde{\delta} t$. Then, the de-spreading result is given by

$$
\widehat{\mathbf{w}}^{\mathcal{T}}=\frac{1}{N_{\mathrm{s}}} \sum_{i=0}^{N_{\mathrm{s}}-1} \tilde{c}_{i} \tilde{\mathbf{r}}^{(i)}
$$

where $\tilde{c}_{i}$ is the same \pm 1 code adopted at tag side. Such $\widehat{\mathbf{w}}^{\mathcal{T}}$ is an estimate of $\mathbf{w}^{\mathcal{T}}=w^{\mathcal{T}}(n \tilde{\delta} t-\tau)$ for $n=1, \ldots, N_{\mathrm{p}}$. Since the tag code used at receiver is balanced (i.e., it is composed of the same number of +1 and -1 ) the result of such an operation is the cancellation of the clutter component $r^{\mathcal{S}}(t)$ which is constant between subsequent frames. Differently, the tag antenna mode component, which is modulated by the same code at tag side, grows and presents a process gain $N_{\mathrm{s}}$, that is, the noise level on the measured useful backscattered component is reduced of a factor $N_{\mathrm{s}}$ thanks to its lack of correlation among different frames. This is fundamental due to the very small contribution coming from the two-hop propagation mechanism, thus enabling the signal characterization also in low SNR conditions, differently from traditional frequency-domain measurements. Such a principle enabling the clutter cancellation and the SNR improvement is depicted in Fig. 10. This technique enables the cancellation of the clutter without resorting to classical approaches adopted in radar solutions, where a first estimation of the background environmental response is collected, then subtracting this data from the actual response. Moreover, no delay lines have to be adopted at tag side to physically separate the antenna mode response from the structural mode response, since the latter is cancelled out as the rest of the background response coming from the environment. Finally, differently from techniques employed on radar of chipless RFID, multiple tags could be also measured and characterized at the same time (or detected/demodulated in practical realizations of the systems), thanks to the possibility of discriminating the signals enabled by the coding scheme. 
The described de-spreading principle has been then improved thanks to the operated oversampling. In fact, we considered for each period of duration $T_{\mathrm{p}}$ the cross-correlation between the first pulse, that is $r(t)$ for $t \in\left[0, T_{\mathrm{p}}\right]$ (i.e., the channel response to the transmitted pulse for $i=0$ ) and the generic $i$ th channel response, that is, $r(t)$ for $t \in\left[i T_{\mathrm{p}},(i+\right.$ 1) $T_{\mathrm{p}}$ ]; since, as anticipated in Sec. III we have $r(t) \approx r^{\mathcal{S}}(t)$, the time instant corresponding to the cross-correlation peak can be adopted instead of the generic $i T_{\mathrm{p}}$ to cut the $i$ th frame and perform the de-spreading. Specifically, $N_{\mathrm{s}}$ timealigned frames, denoted with $\mathbf{u}^{(i)}$, with $i=0, \ldots, N_{\mathrm{s}}-1$, are constructed from $\tilde{\mathbf{r}}$ according to

$$
\mathbf{u}^{(i)}=\tilde{\mathbf{r}}\left(\left(\left(i+\Delta^{(i)}\right) \cdot N_{\mathrm{p}}+1\right):\left(\left(i+\Delta^{(i)}\right) \cdot N_{\mathrm{p}}+N_{\mathrm{p}}\right)\right)
$$

where $\Delta^{(i)}=\hat{n}-1$ and $\hat{n}$ is the index corresponding to the maximum of the cross-correlation vector, indexed by $n=$ $1, \ldots, N_{\mathrm{p}}$

$$
\max _{n=1 \ldots, N_{\mathrm{p}}} \operatorname{IFFT}\left[\operatorname{FFT}\left[\tilde{\mathbf{r}}^{(0)}\right] \odot \operatorname{FFT}\left[\tilde{\mathbf{r}}^{(i)}\right]^{*}\right] \quad i=0, \ldots N_{\mathrm{s}}-1
$$

having indicated with $\odot$ the point wise product and with $(\cdot)^{*}$ the conjugate. These time-aligned frames $\mathbf{u}^{(i)}$ are then adopted in the de-spreading (10) instead of $\tilde{\mathbf{r}}^{(i)}$. This allows compensating the small difference between the oscillator of the AWG, driving the transmission instants of the pulses, and that of the oscilloscope, driving the ADC, and correcting the sampling instant drift. Again, as showed in (12), in order to maximize the performance, the $N_{\mathrm{s}}-1$ cross-correlations are operated in the frequency domain by considering the multiplication of the FFT of the two involved frames.

\section{Code Acquisition}

Unfortunately, the offset (phase) of the tag code to be used for the de-spreading is not known since the tag is not synchronous with the trigger driving the oscilloscope acquisition. Then, a code acquisition stage has been implemented as a signal processing block in order to search for the best code offset in the de-spreading. Specifically, $N_{\mathrm{pc}}$ code shifts of the code adopted for the de-spreading are considered. For each code shift a de-spreading is operated and the overall energy of the obtained waveform of length $T_{\mathrm{p}}$ is measured (see Fig. 6). Finally, a decision in favor of the code shift resulting in the maximum output energy is taken, and the corresponding code is adopted for de-spreading. Formalizing, we have

$$
\widehat{m}=\underset{m=1, \ldots, N_{\mathrm{pc}}}{\operatorname{argmax}}\left\|\frac{1}{N_{\mathrm{s}}} \sum_{i=0}^{N_{\mathrm{s}}-1} \tilde{c}_{i+m} \mathbf{u}^{(i)}\right\|^{2}
$$

and in (10) the code $\tilde{c}_{i+\widehat{m}}$ is adopted instead of $\tilde{c}_{i}$.

\section{Example of Processing Result}

The result of such a de-spreading operation is shown in Fig. 11. As it is possible to notice, the tag signal TOA is different from the clutter signal TOA of Fig. 8 (which starts at the beginning of the windows with the antenna coupling since $\tau^{\prime} \approx 0$ in the considered quasi-monostatic configuration) due to the distance between the transmitting/receiving

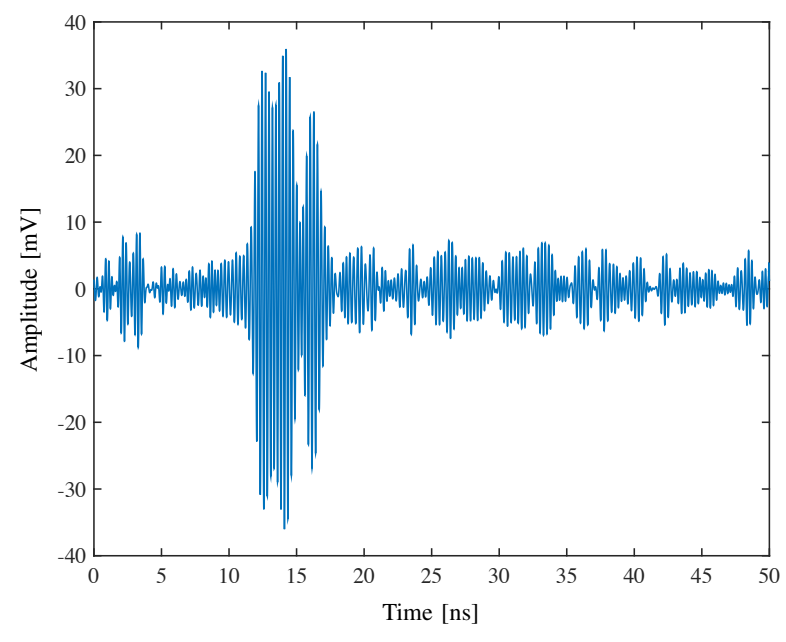

Fig. 11. Processing result (estimated tag antenna component) for the signal reported in Fig. 8

antennas and the tag antenna. The analysis of the signal TOA, which can be realized with different techniques [10], enables the estimation of the reader-tag distance, thus allowing the possibility of localizing the tag by fusing several ranging data, or range plus angle-of-arrival (AOA) information. Moreover, the channel profile of the tag response is different from the overall response of Fig. 8 due to particular tag position in the environment. Finally, as expected, the signal amplitude is significantly lower of that visible in Fig. [8 due to the twohop propagation mechanism intrinsic of backscattering. The duration of the signal reported in Fig. 10 is $50 \mathrm{~ns}$, that is, the duration of a frame (PRP). In fact, thanks to the accumulation and de-spreading process, several portions of the received signal are summed up in order to obtain the raw response to a single pulse experiencing a proper processing gain. In Fig. 11] and in the subsequent results, the de-spreading result is always divided by the number of accumulated pulses $N_{\mathrm{s}}$ composing a symbol, according to (10), in order to read, in the vertical axis, the amplitude in Volt of the backscattered pulse (including amplifiers and antenna gain). In the following section experimental results with different tag antennas will be presented and the effect of the processing parameters discussed.

\section{EXPERIMENTAL RESULTS}

In this section experimental results are proposed. The capability offered by the presented setup of extracting the antenna mode backscattered component of wideband antennas will be discussed. Then, the effect of the choices in terms of tags' spreading codes and signal processing parameters will be characterized.

\section{A. General Measurement and Processing Results}

In order to verify the effect of the processing scheme, in Fig. 12(a) a frame of the original signal collected by the oscilloscope is showed (in orange). At first glance, all the $N_{\mathrm{s}}$ frames look identical due to the dominant amplitude of the 


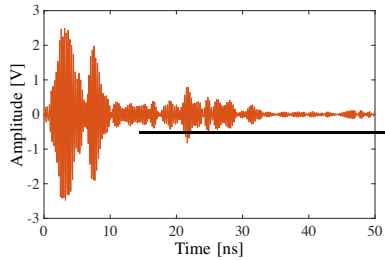

(a) Received signal (raw)

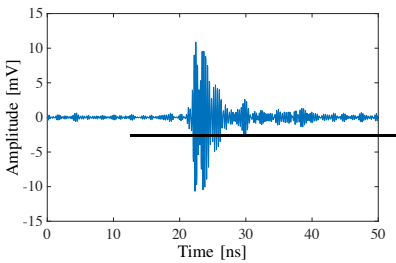

(c) Tag at $300 \mathrm{~cm}$

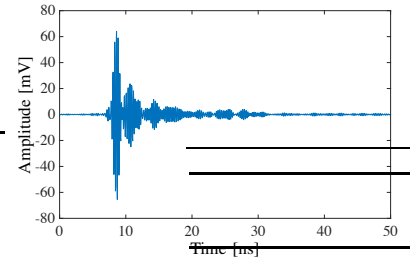

(b) Tag at $90 \mathrm{~cm}$

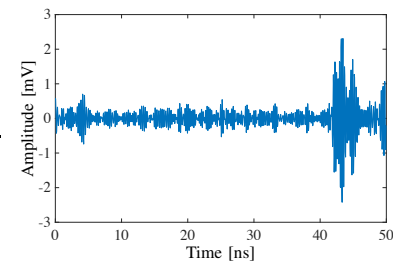

(d) Tag at $600 \mathrm{~cm}$
Fig. 12. Example of measurements: tags backscattering at different distances from the transmitting/receiving antennas.

clutter component. Specifically, the amplitude of the acquired signal is around $5 \mathrm{Vpp}$. Differently, the 3 following pictures of Fig. 12 show the processing results when a tag is present at $0.9 \mathrm{~m}, 3 \mathrm{~m}$ and $6 \mathrm{~m}$ of reader-tag distance, respectively. In these cases, the amplitude of the signal, now composed of the antenna mode component and noise only, is significantly lower (around $120 \mathrm{mVpp}, 20 \mathrm{mVpp}$ and $4 \mathrm{mVpp}$, respectively, scaling with the reader-tag distance). Moreover, it is possible to appreciate how the starting point of the pulse (i.e., the position of the first path of the backscattered signal) is a function of the reader-tag distance due to the signal propagation in air. Notice that the noise floor, clearly visible in Fig. 12(d) before the signal TOA, comes from the accumulation of all the $N_{\mathrm{s}}=10000$ received pulses enabled by the 12.5 Msamples memory of oscilloscope (2). The adoption of a sampler with smaller amount of memory would lead to the accumulation of a smaller number of pulses, thus in a larger noise floor. This limits the capability of revealing the tag signal from the noise, which translates in a shorter maximum reader-tag distance where the measure can be realized.

The effectiveness of the adopted processing scheme in extracting the antenna mode component is confirmed in Fig. 13. In this case, again, in the orange picture $(13$ (a)) a frame of the signal acquired by the oscilloscope is reported. In the following blue picture, the processing result when a tag is active (i.e., switching its backscatter modulator between open and short loads) is presented (Fig. 13 (b)) 10 As expected, a pulse can be found at a time instant corresponding to the given reader-tag distance. Then, the processing results in particular conditions follow. In Fig. 13 (c) the outcome obtained with the micro controller not switching is shown; in this case the antenna mode backscattering component is composed of always the same open or short response, depending on the switch status (now permanent). Thus, after the processing, only noise if present in output, as it can be seen in the

${ }^{10}$ Notice that different antennas were considered with respect to results of Fig. 12 leading to different amplitude of the backscattered signal.

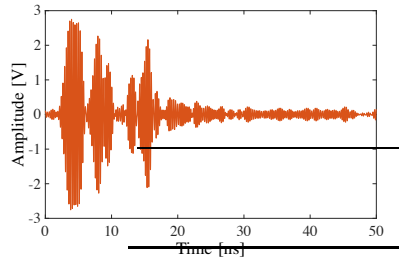

(a) Received signal (raw)

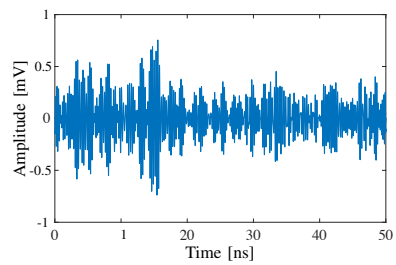

(c) $\mu \mathrm{C}$ off

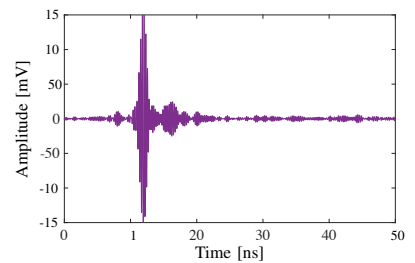

(e) $\mu \mathrm{C}$ on (open/matched loads)

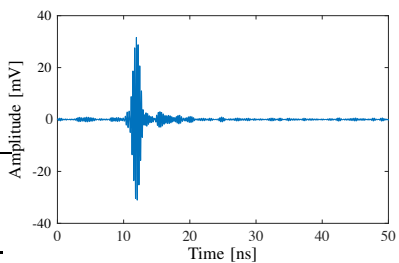

(b) $\mu \mathrm{C}$ on (open/short loads)

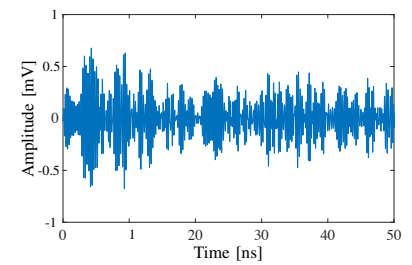

(d) Matched load only

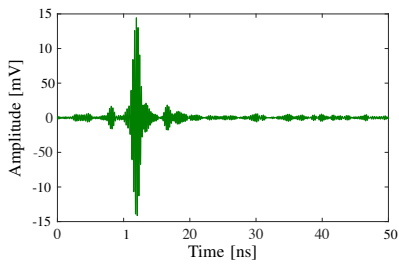

(f) $\mu \mathrm{C}$ on (short/matched loads)
Fig. 13. Example of measurements: de-spreading results in different operating conditions.

figure, since $N_{\mathrm{S}} / 2$ antenna mode responses are cancelled out by the other $N_{\mathrm{s}} / 2$ identical responses due to the multiplication for the balanced code at receiver side (as happens for the clutter component). The same behavior is obtained when the tag antenna is not loaded with open/short loads, but with a matched load (i.e., $50 \Omega$ ), as reported in Fig. 13 d). In this case the tag response is composed of the structural mode response only, and no antenna mode components depending on the unmatched loads are backscattered. Then, the despreading result is again composed of noise only. The last two results offered in Fig. 13 report a tag with its switch ports connected, instead to the couple of open/short loads, with a couple open/matched loads (Fig. 13(d)) or short/matched loads (Fig. 13 (e)), respectively. Notice that, also with this tag configuration, the antenna mode component is present at the output of the de-spreading. In fact, again, the clutter cancels out and the $N_{\mathrm{s}} / 2$ channel responses due to the tag antenna mode component sum up coherently. However, since the antenna mode signal is not composed of antipodal components for the two load conditions (in fact, the antenna mode will be present only in one of the two switch states, while the structural mode only will be present in the other switch status), the obtained signal amplitude is halved with respect to the ideal case of Fig. 13 b) (i.e., $30 \mathrm{mVpp}$ instead of $60 \mathrm{mVpp}$ ).

A more detailed analysis of the single responses related to the open or short loads at tag side is reported in Fig. 14 These results have been obtained according to the following measurement setup. The two switch ports were connected to a couple of delay-matched transmission lines. Then, one port 


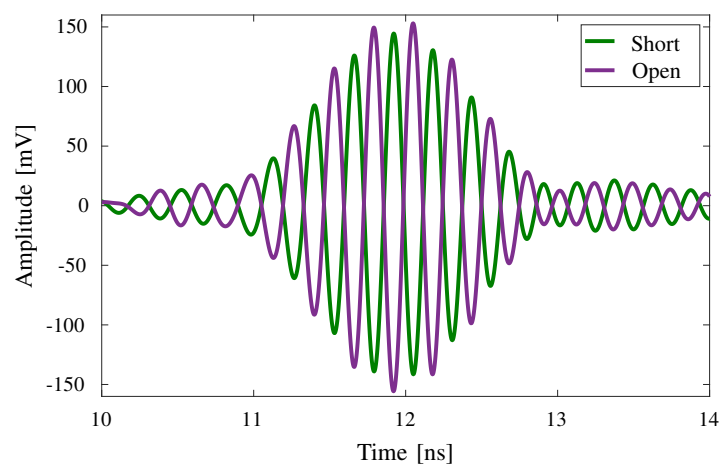

Fig. 14. Measured tag open circuit and short circuit responses.

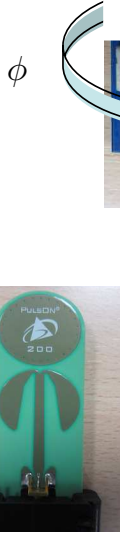

(a)

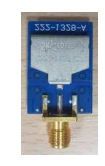

(b)
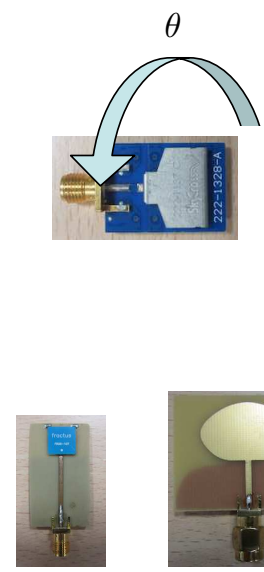

(c)

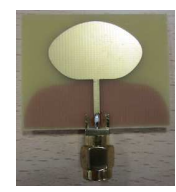

(d)
Fig. 15. Commercial UWB antennas considered for tag measurements: (a) Time Domain antenna, (b) SkyCross antenna, (c) Fractus antenna, (d) Decawave antenna.

of the transmission line was connected to a matched load (i.e., $50 \Omega$ ), while the other to the short load. A measurement was taken according to this scheme. The result of the de-spreading is, in this case, a pulse given by the difference of the response between short load and matched load. Since the response in the matched load case is composed of structural mode only, we obtained the signal response related to the short load case. By repeating the same procedure manually changing the load between short and open 11 the response related to the open load case was obtained. As it it possible to see from Fig. 14. the two responses have a $180^{\circ}$ phase difference, as expected (only the 1 st path is reported).

\section{B. Backscattering Characteristics of Commercial Antennas}

In this section, test concerning different commercial antennas used in backscattering are presented. The considered antennas are reported in Fig. 15 Specifically, they are:

- Broadspec antenna by Time Domain Corp.;

- SMT-3T01OM antenna by Skycross;

- FR05-107 antenna by Fractus;

${ }^{11}$ The delay-matched transmission line allows changing the load without moving the tag antenna which could result in an unpredictable different response.

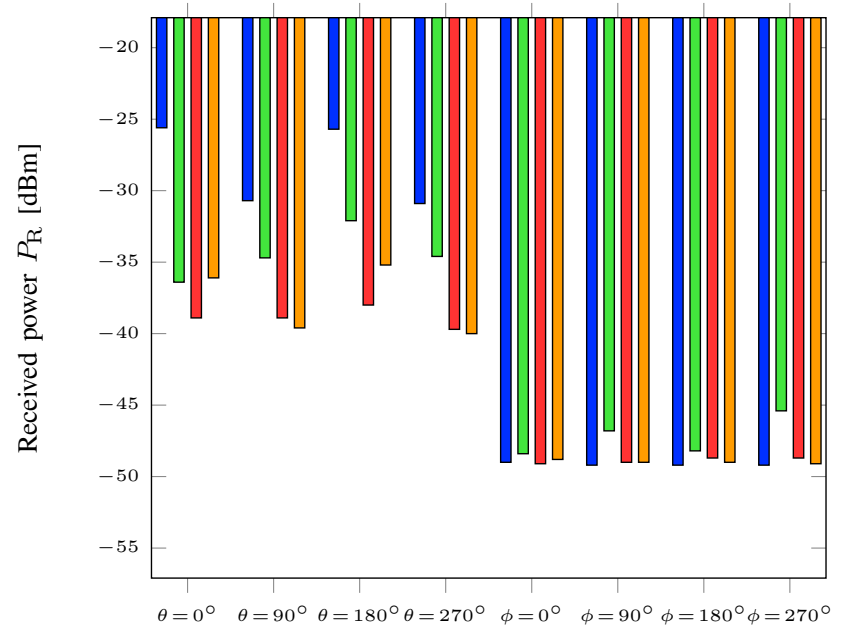

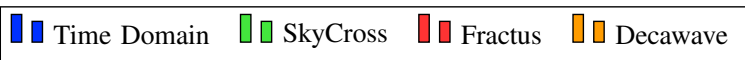

Fig. 16. Received power $P_{\mathrm{R}}$ from commercial UWB antennas in backscattering.

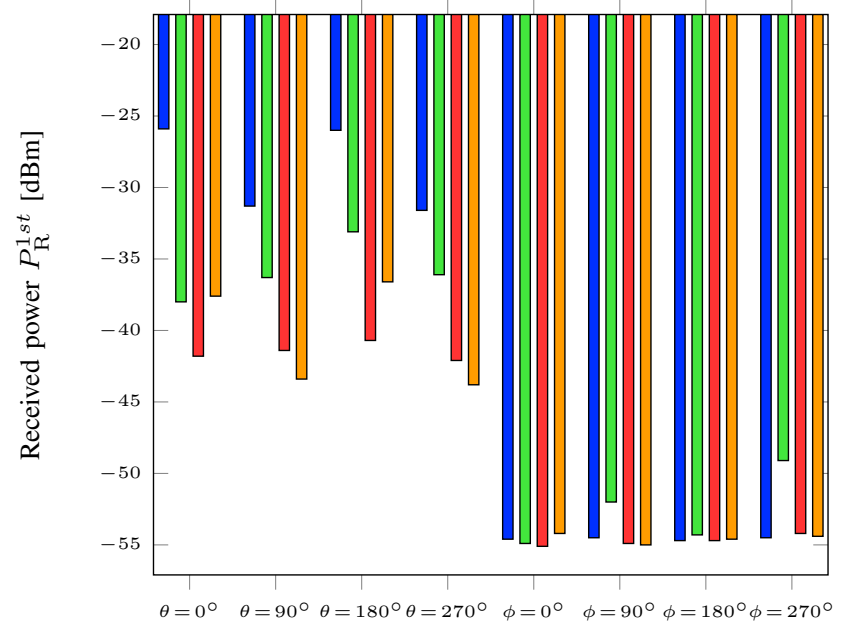

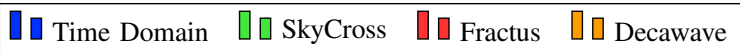

Fig. 17. Received power $P_{\mathrm{R}}^{1 s t}$ from commercial UWB antennas in backscattering.

- WB002 antenna by Decawave.

All the considered antennas have a dipole-like radiation pattern and present linear polarization. They were tested considering 4 different rotations in the vertical plane and 4 different rotations in the horizontal plane (Fig. 15). In all the cases, the reader transmitting and receiving antennas were kept horizontal then resulting in 4 measurements with the same polarization for reader and tag (rotating along $\theta$ ), and 4 measurements in cross-polarization (rotating along $\phi$ ).

Figure 16 shows the received power for the 4 antennas 12 considering the 4 cases of accorded polarization with respect to the reader (first 4 groups of histograms) and the 4 cases of cross-polarization (following 4 groups of histograms). The

\footnotetext{
${ }^{12}$ The presented values include the power gain due to the receiver amplifiers.
} 


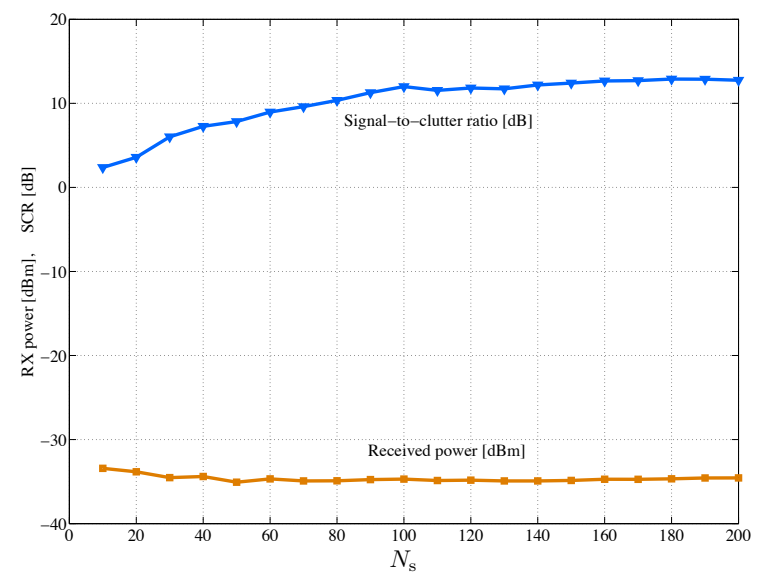

Fig. 18. Impact of $N_{\mathrm{S}}$ on the estimated received power and on the SCR.

received power $P_{\mathrm{R}}$ was computed as the ratio between the received energy in a frame and the frame duration, that is

$$
P_{\mathrm{R}}=\frac{1}{T_{\mathrm{p}} R} \int_{0}^{T_{\mathrm{p}}}\left[\widehat{w}^{\mathcal{T}}(t-\tau)\right]^{2} d t
$$

where $R=50 \Omega$ is the load impedance. It is possible to appreciate that the receiver power in the case of crosspolarization is around $10 \mathrm{~dB}$ lower than the corresponding case with accorded polarization. In this case, in fact, the energy comes at most from noise and scattered multipath components. Differently, with the first 4 groups of histograms, it is possible to appreciate the difference in terms of received power related to the response of the 4 antennas considering the different rotations.

In Fig. 177 the same results of Fig. 16 are offered, but considering the first path only for the received power calculation. In fact, in this case, the received power was obtained as the ratio between the energy measured integrating the receiving signal around its peak (corresponding to the first path in lineof-sight (LOS)) for a duration equal to the pulse width, and the frame duration, that is

$$
P_{\mathrm{R}}^{1 s t}=\frac{1}{T_{\mathrm{p}} R} \int_{\hat{\tau}-T_{\mathrm{w}}}^{\hat{\tau}+T_{\mathrm{w}}}\left[\widehat{w}^{\mathcal{T}}(t-\tau)\right]^{2} d t
$$

where $\hat{\tau}$ is the received signal peak. Due to this fact, the received power is lower since, now, it is less affected by the multipath components.

\section{Signal Processing Impact}

In this section, the effects of the different choices in terms of signal processing parameters are investigated. In particular, the effects of the number of accumulated pulses $N_{\mathrm{s}}$ and of the oversampling factor $\mathrm{OF}$ are characterized. As figures of merit, the estimated received power $P_{\mathrm{R}}$ from the tag backscattering, and the signal-to-clutter ratio (SCR) are considered. The estimated received power should be, theoretically, independent of both $N_{\mathrm{s}}$ and OF since it is related to the tag backscattering capability only. However, since it is measured after the processing blocks discussed in the previous sections, it can (a) Received signal $-N_{\mathrm{s}}=20$

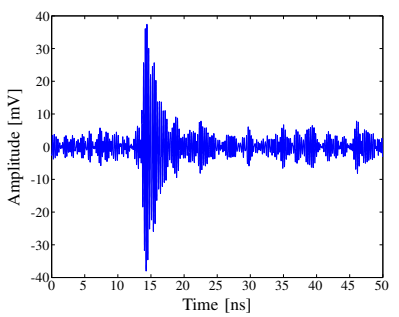

(c) Received signal $-N_{\mathrm{s}}=200$

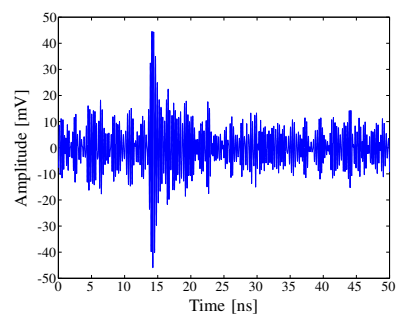

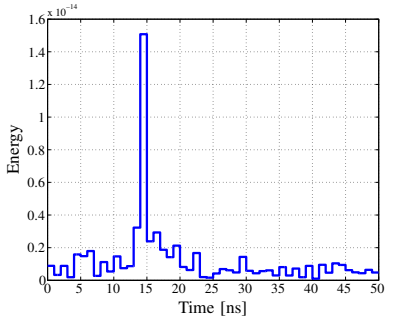

(b) Energy profile $-N_{\mathrm{S}}=20$

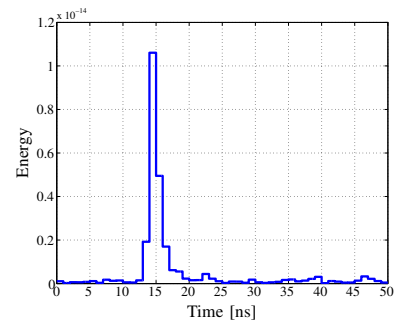

(d) Energy profile $-N_{\mathrm{S}}=200$
Fig. 19. Example of impact of the number of pulses $N_{\mathrm{s}}$ on the received waveform.

be affected by some choices of the parameters. Results were obtained with oscilloscope (1). For further convenience, the received signal of length $T_{\mathrm{p}}$ is divided in $N_{\text {bin }}$ energy bins, each related to the energy of the waveform in a particular time interval. This is done in line with the processing frequency exploited for signal TOA estimation to enable tag localization. In fact, the energy profile enables an easier characterization of the instant where the signal starts among the noise, by keeping the general complexity low thanks to the implementation at sub-Nyquist rate [10]. In particular we have

$$
\mathbf{e}(m)=\int_{(m-1) T_{\mathrm{ED}}}^{m T_{\mathrm{ED}}}[\widehat{w}(t-\tau)]^{2} d t
$$

with $m=1,2, \ldots, N_{\text {bin }}$ and where $N_{\text {bin }}=\left\lfloor T_{\mathrm{p}} / T_{\mathrm{ED}}\right\rfloor$ represents the number of integration bins in which each frame is divided in for an energy detector integration time $T_{\mathrm{ED}}$. Specifically, since $T_{\mathrm{p}}=50 \mathrm{~ns}$ in the conducted measurements, $N_{\text {bin }}=50$ bins of $T_{\mathrm{ED}}=1 \mathrm{~ns}$ width were considered. Starting from the energy profile e composed by the set of all the bins, the SCR was defined as the ratio between the energy related to the largest bin (corresponding to the signal TOA, i.e., the first path of the tag backscattered signal) and the energy of the first 5 bins, usually containing noise and residuals of the direct TX-RX antennas coupling, which is the largest clutter component, that is, the most difficult to cancel out for the processing scheme. Formally, we have

$$
\mathrm{SCR} \triangleq \frac{\max _{n} \mathbf{e}(n)}{\sum_{i=1}^{5} \mathbf{e}(i)} .
$$

Figure 18 shows how the estimated backscattered power and SCR behave as a function of $N_{\mathrm{s}}$. It is possible to notice that, with small $N_{\mathrm{s}}$ (few accumulate pulses) the SCR is lower due to the presence of more noise and clutter residual in the signal. The estimated power is robust with respect to $N_{\mathrm{s}}$. In general, 


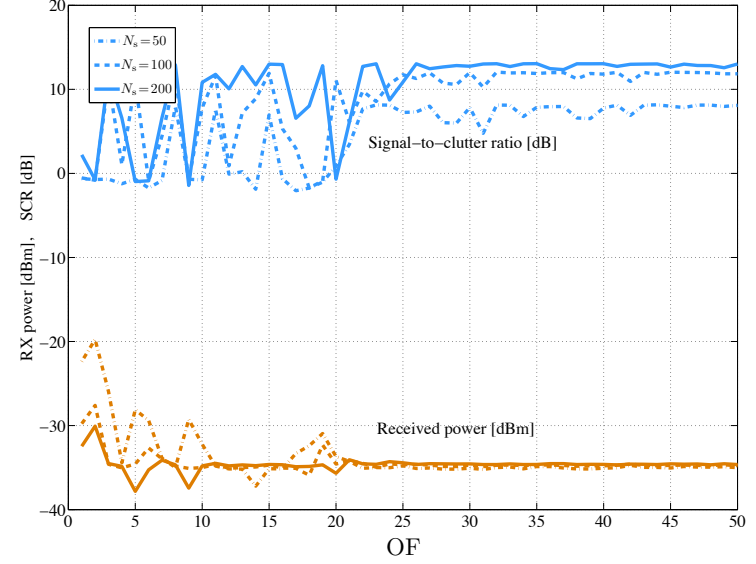

Fig. 20. Impact of OF on the estimated received power and on the SCR.

considering $N_{\mathrm{s}}>100$ both the results are very stable and the processing output can be considered reliable. Examples of the received signals and energy profiles for two different values of $N_{\mathrm{s}}$ are given in Fig. 19] It is possible to see that with $N_{\mathrm{s}}=20$ the signal is more corrupted by the noise but, from the energy profile on the right, it is clear the first path of the tag backscattered signal starting from bin 15 (i.e., TOA of $15 \mathrm{~ns}$ ). Differently, adopting $N_{\mathrm{s}}=200$, the noise level is much lower than in the previous case and the energy profile results very clean, leading to a more reliable detection and correct TOA estimation for practical applications.

In Fig. 20] the effect of the oversampling is shown. As before, the estimated power and the SCR are considered as figures of merit as a function of the oversampling factor OF. Results are given for three different values of $N_{\mathrm{s}}$, that are, $N_{\mathrm{s}}=50, N_{\mathrm{s}}=100$ and $N_{\mathrm{s}}=200$. Looking at the figure, it is possible to appreciate as an oversampling factor $\mathrm{OF}>25$ is necessary to obtain reliable measurement results. In fact, if $\mathrm{OF}$ is too low we experience oscillations in the SCR and a potential wrong estimation of the received backscattered power from the tag. These facts are due to the presence of clutter residuals on the received waveform, not properly cancelled out by the processing scheme. Differently, when a proper value of $\mathrm{OF}$ is considered, the results are stable and the processing output can be considered reliable. Practical examples of these effects can be found in Fig. 21. In the upper part the received waveform (after the processing) and the related energy profile are shown without considering any form of oversampling. Differently, the same signals are reported in the bottom part of the figure considering $\mathrm{OF}=25$ in the processing chain. It is clear as, without oversampling, large clutter residuals are present. These clutter residuals impact the waveform in time instants before the true signal TOA, then can lead to errors in TOA estimation.

\section{Code Effects}

The following results consider the effect of the spreading code adopted at tag side on the measurement results. In particular it is shown how the rate of switching between (a) Received signal - $\mathrm{OF}=1$

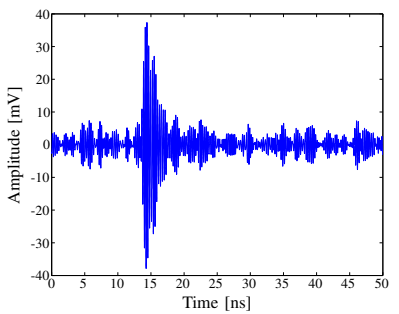

(c) Received signal - $\mathrm{OF}=25$

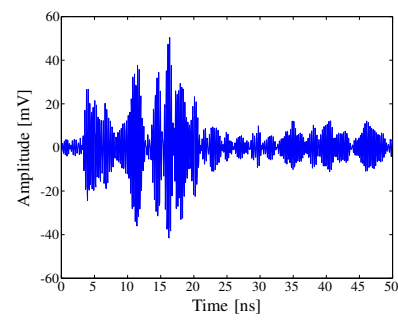

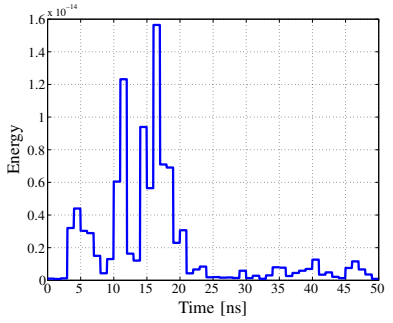

(b) Energy profile - $\mathrm{OF}=1$

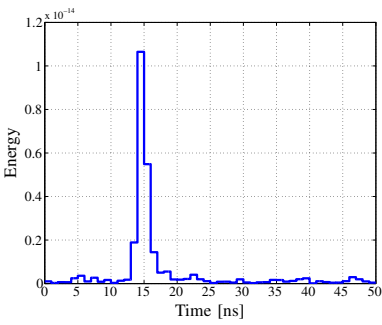

(d) Energy profile $-\mathrm{OF}=25$
Fig. 21. Example of impact of the oversampling factor $\mathrm{OF}$ on the received waveform.

open and short circuit impacts the performance. Histograms in Fig. 22 show the estimated received power and SCR for different switching rates, that is, different values of the pulses per chip $N_{\mathrm{pc}}$. Specifically, $N_{\mathrm{pc}}=5, N_{\mathrm{pc}}=10, N_{\mathrm{pc}}=20$, $N_{\mathrm{pc}}=50$ and $N_{\mathrm{pc}}=100$ are considered, always with $N_{\mathrm{s}}=200$. The general behavior shows a decreasing of the SCR if $N_{\mathrm{pc}}$ increases. At the same manner, the estimated power slightly increases. The decreasing of the SCR can be explained with the progressive born and growth of clutter residuals not properly cancelled out by the processing scheme. In fact, when the tag switches frequently between open and short loads, to consecutive responses are immediately summed up, after the multiplication for the expected code value, so that the clutter cancels out precisely. Differently, when a low switching rate between open and short is considered, many responses are accumulated and are summed up. Then, if few differences are present between the responses due to timedistant pulses presenting non-idealities (e.g., the first pulse related to open circuit and the 101th pulse related to short circuit) the clutter cancelation could not be perfect, then increasing the SCR. This fact is confirmed by Fig. 23 where the received signals and energy profile are shown considering $N_{\mathrm{pc}}=5$ (top) and $N_{\mathrm{pc}}=100$ (bottom). In the case of $N_{\mathrm{pc}}=5$ the tag signal and energy profile are very clean. Differently, when $N_{\mathrm{pc}}=100$, a clutter residual arises in the first part of the received waveform, mainly due to the strong TX-RX antenna coupling not properly cancelled out by the processing algorithm. If this strong component at the beginning of the signal cannot be properly cancelled out with the processing, a simple yet effective way is the time-gating of the waveform, which consists in the cancellation of the initial portion of the resulting signal where such a contribution is present. In practical applications, considering a monostatic configuration, this time-gating produces a region around the reader where the tag cannot be detected, since also the corresponding antenna 


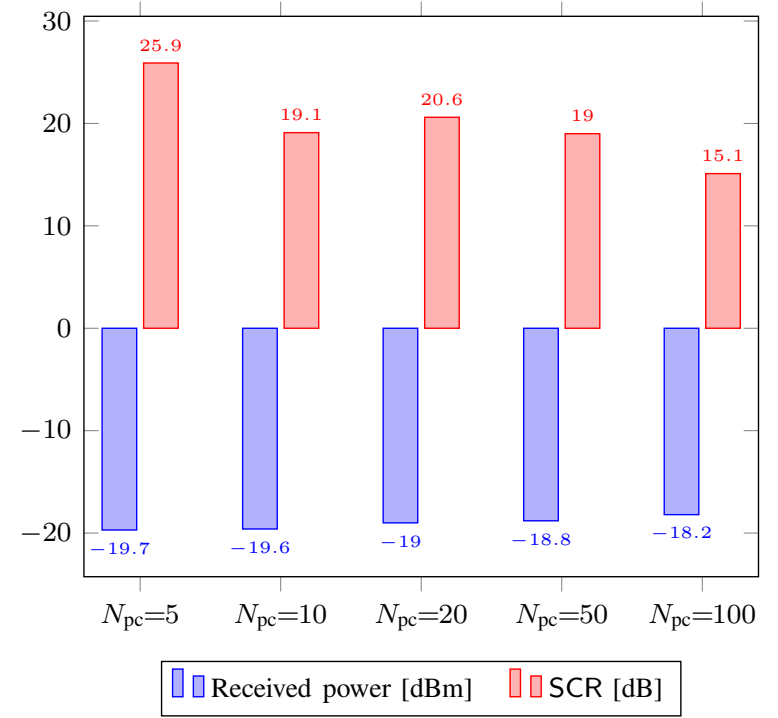

Fig. 22. Impact of the code length on the estimated received power and on the SCR.

mode component would be canceled. Notice that this not-ideal clutter cancellation translates into a small energy bins before the tag signal TOA then, if the detection/estimation thresholds are properly designed, it does not impact the performance, especially for small reader-tag distance (i.e., large tag signal SNR).

\section{CONCLUSION}

This paper proposed a time-domain measurement setup for the characterization of signals backscattered by wideband RFID tags. An ad-hoc signal processing scheme for the extraction of the antenna mode component and the cancellation of the clutter has been described. Beside the realization of measurements, the proposed techniques can drive the realization of receivers for the acquisition of wideband backscatter signals. Results showed how it is possible to isolate the antenna mode component, although buried by the clutter, starting from timedomain samples, without requiring the tag and the environment to be static for a certain amount of time and without the adoption of delay lines at tag side, as usually required by other techniques. The experimentation provided insights on the achievable results, highlighting how the choices of signal processing parameters affect the measurement performance.

\section{ACKNOLEDGMENT}

Authors would like to thank M. Filippi, A. Romani, M. Del Prete, A. Guerra, F. Guidi, S. Bartoletti, A. Conti and M. Chiani for the cooperation in the setting up and measurements.

\section{REFERENCES}

[1] K. Finkenzeller, RFID Handbook: Fundamentals and Applications in Contactless Smart Cards and Identification., 3rd ed. Wiley, 2010.

[2] EPCGlobal, "Class 1 generation 2 UHF air interface protocol standard v.1.0.9," 2005.

[3] A. R. J. Ruiz and F. S. Granja, "Comparing ubisense, bespoon, and decawave UWB location systems: Indoor performance analysis," IEEE Trans. Instrum. Meas., vol. 66, no. 8, pp. 2106-2117, Aug. 2017. (a) Received signal - $N_{\mathrm{pc}}=5$

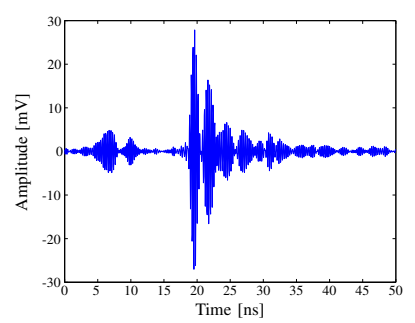

(c) Received signal - $N_{\mathrm{pc}}=100$

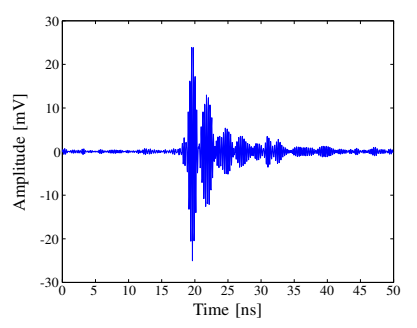

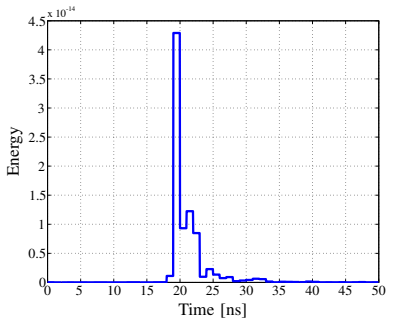

(b) Energy profile $-N_{\mathrm{pc}}=5$

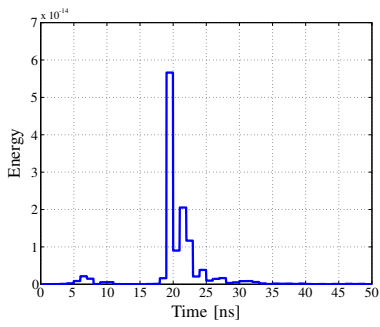

(d) Energy profile $-N_{\mathrm{pc}}=100$
Fig. 23. Example of impact of the code length on the received waveform.

[4] L. Ni, D. Zhang, and M. Souryal, "RFID-based localization and tracking technologies," IEEE Wireless Commun., vol. 18, no. 2, pp. 45-51, Apr. 2011.

[5] D. Dardari et al., "Ultrawide bandwidth RFID: The next generation?" Proc. IEEE, vol. 98, no. 9, pp. 1570-1582, Sep. 2010.

[6] — "The future of ultra-wideband localization in RFID," in IEEE Int. Conf. RFID, Orlando, FL, May 2016, pp. 1-7.

[7] N. Decarli, F. Guidi, and D. Dardari, "Passive UWB RFID for tag localization: Architectures and design," IEEE Sensors J., vol. 16, no. 5, pp. 1385-1397, Mar. 2016.

[8] D. Girbau et al., "Passive wireless temperature sensor based on timecoded UWB chipless RFID tags," IEEE Trans. Microwave Theory Tech., vol. 60, no. 11, pp. 3623-3632, Nov. 2012.

[9] A. Ramos, A. Lazaro, and D. Girbau, "Semi-passive time-domain UWB RFID system," IEEE Trans. Microwave Theory Tech., vol. 61, no. 4, pp. 1700-1708, Apr. 2013.

[10] D. Dardari et al., "Ranging with ultrawide bandwidth signals in multipath environments," Proc. IEEE, vol. 97, no. 2, pp. 404-426, Feb. 2009.

[11] Y. Shen and M. Z. Win, "Fundamental limits of wideband localization Part I: A general framework," IEEE Trans. Inf. Theory, vol. 56, no. 10, pp. 4956-4980, Oct. 2010.

[12] J. Lai et al., "Wireless sensing of human respiratory parameters by lowpower ultrawideband impulse radio radar," IEEE Trans. Instrum. Meas., vol. 60, no. 3, pp. 928-938, Mar. 2011.

[13] E. Piuzzi et al., "Complex radar cross section measurements of the human body for breath-activity monitoring applications," IEEE Trans. Instrum. Meas., vol. 64, no. 8, pp. 2247-2258, Aug. 2015.

[14] J. C. Y. Lai et al., "Wireless sensing of human respiratory parameters by low-power ultrawideband impulse radio radar," IEEE Trans. Instrum. Meas., vol. 60, no. 3, pp. 928-938, Mar. 2011.

[15] A. Ramos et al., "Time-domain measurement of time-coded UWB chipless RFID tags," Progress in Electromagnetics Research, vol. 116, pp. 313-331, 2011.

[16] S. Hu et al., "Study of a uniplanar monopole antenna for passive chipless UWB-RFID localization system," IEEE Trans. Antennas Propag., vol. 58, no. 2, pp. 271-278, Feb. 2010.

[17] S. Hu, C. Law, and W. Dou, "A balloon-shaped monopole antenna for passive UWB-RFID tag applications," IEEE Antennas Wireless Propag. Lett., vol. 7, pp. 366-368, 2008.

[18] F. Guidi et al., "Analysis of UWB tag backscattering and its impact on the detection coverage," IEEE Trans. Antennas Propag., vol. 62, no. 8, pp. 4292-4303, Aug. 2014

[19] R. D'Errico, "An indoor backscattering channel characterization for UWB passive RFID applications," in European Conf. on Antennas and Propag. (EuCAP), Prague, Czech Republic, Mar. 2012, pp. 1169-1173.

[20] R. D'Errico, J. Keignart, and L. Rudant, "Characterization of UWB backscattering propagation for passive tags identification and localiza- 
tion," in European Conf. on Antennas and Propag. (EuCAP), Gothenburg, Sweden, Apr. 2013, pp. 1909-1913.

[21] A. Sibille et al., "Channel modeling for backscattering based UWB tags in a RTLS system with multiple readers," in Proc. European Conf. on Antennas and Propag. (EuCAP), Gothenburg, Sweden, Apr. 2013, pp. 3022-3026.

[22] F. Guidi et al., "Detection of multiple tags based on impulsive backscattered signals," IEEE Trans. Commun., vol. 62, no. 11, pp. 3918-3930, Nov. 2014.

[23] N. Decarli, F. Guidi, and D. Dardari, "A novel joint RFID and radar sensor network for passive localization: Design and performance bounds," IEEE J. Sel. Topics Signal Process., vol. 8, no. 1, pp. 80-95, Feb. 2014.

[24] J. Kimionis, A. Bletsas, and J. N. Sahalos, "Increased range bistatic scatter radio," IEEE Trans. Commun., vol. 62, no. 3, pp. 1091-1104, Mar. 2014.

[25] "IEEE standard for information technology - telecommunications and information exchange between systems - local and metropolitan area networks - specific requirement part 15.4: Wireless medium access control (MAC) and physical layer (PHY) specifications for low-rate wireless personal area networks (WPANs)," IEEE Std 802.15.4a-2007 (Amendment to IEEE Std 802.15.4-2006), pp. 1-203, 2007. 